\title{
ORIGINAL ARTICLE \\ Movement repetitions in physical and occupational therapy during spinal cord injury rehabilitation
}

\author{
D Zbogar ${ }^{1,2,3}$, JJ Eng ${ }^{1,2,3}$, WC Miller ${ }^{1,2,4}$, AV Krassioukov ${ }^{2,5}$ and MC Verrier ${ }^{6,7}$
}

Study design: Longitudinal observational study.

Objective: To quantify the amount of upper- and lower-extremity movement repetitions (that is, voluntary movements as part of a functional task or specific motion) occurring during inpatient spinal cord injury (SCI), physical (PT) and occupational therapy (OT), and examine changes over the inpatient rehabilitation stay.

Setting: Two stand-alone inpatient $\mathrm{SCl}$ rehabilitation centers.

Methods: Participants: A total of 103 patients were recruited through consecutive admissions to SCl rehabilitation. Interventions: Trained assistants observed therapy sessions and obtained clinical outcome measures in the second week following admission and in the second to last week before discharge. Main outcome measures: PT and OT time, upper- and lower-extremity repetitions and changes in these outcomes over the course of rehabilitation stay.

Results: We observed 561 PT and 347 OT sessions. Therapeutic time comprised two-thirds of total therapy time. Summed over PT and OT, the median upper-extremity repetitions in patients with paraplegia were 7 repetitions and in patients with tetraplegia, 42 repetitions. Lower-extremity repetitions and steps primarily occurred in ambulatory patients and amounted to 218 and 115 , respectively (summed over PT and OT sessions at discharge). Wilcoxon-signed rank tests revealed that most repetition variables did not change significantly over the inpatient rehabilitation stay. In contrast, clinical outcomes for the arm and leg improved over this time period.

Conclusions: Repetitions of upper- and lower-extremity movements are markedly low during PT and OT sessions. Despite improvements in clinical outcomes, there was no significant increase in movement repetitions over the course of inpatient rehabilitation stay.

Spinal Cord (2017) 55, 172-179; doi:10.1038/sc.2016.129; published online 18 October 2016

\section{INTRODUCTION}

Occupational therapy (OT) and physical therapy (PT) have a central role in the rehabilitation of individuals who have experienced a spinal cord injury (SCI). Over the past several years, quantifying therapy content in SCI rehabilitation has received increasing attention to better understand current practice. The SCIRehab project is a notable comprehensive and recent example, ${ }^{1}$ in which therapists recorded the number of sessions, minutes, activity-specific details and the extent of patient participation in $\mathrm{PT}^{2}$ and $\mathrm{OT}^{3}$ sessions in inpatient rehabilitation. Although studies of content and time spent on activities $^{4-8}$ provide a key component to unraveling the relationship between therapeutic intervention and outcomes, they do not provide an indication of the amount of active therapist-directed movement repetitions during that time, which are important for optimizing neuroplasticity.

Research studies in animals and humans have found that remodeling in the nervous system accompanies the practice of motor tasks after SCI and can facilitate the recovery of locomotor function ${ }^{9}$ and reaching. ${ }^{10}$ In patients with incomplete SCI, rehabilitation therapies such as repetitive upper-extremity movements improve hand function, ${ }^{11,12}$ whereas locomotor training promotes ambulatory recovery. ${ }^{13}$ However, improved locomotor capacity after SCI in the animal literature involves several hundred to over a thousand repetitions ${ }^{14,15}$ with higher doses resulting in improved outcomes. Overall, the animal and human motor-learning literature supports repetitions in the mid-hundreds to thousands to show improvement in upper- or lower-extremity measures. ${ }^{11-15}$ Measuring repetitions is also important as it forms a basis for task-specific practice, as well as muscle conditioning, to ultimately facilitate functional outcomes. For example, repetitions of movements involving the arms may strengthen the arms and help learn correct movements to reduce the increased potential for musculoskeletal injury that results from propelling a manual wheelchair or performing transfers, whether the individual has tetraplegia or paraplegia with neurologically intact upper extremities. Unfortunately, there are no reports that estimate the movement repetitions during human SCI rehabilitation, and thus we do not know whether patients are receiving sufficient repetitions to optimize

${ }^{1}$ Rehabilitation Research Program, Vancouver Coastal Health Research Institute, Vancouver, BC, Canada; ${ }^{2}$ International Collaboration on Repair Discoveries, Vancouver, BC, Canada; ${ }^{3}$ Department of Physical Therapy, Faculty of Medicine, University of British Columbia, Vancouver, BC, Canada; ${ }^{4}$ Department of Occupational Science \& Occupational Therapy, Faculty of Medicine, University of British Columbia, Vancouver, BC, Canada; ${ }^{5}$ Division of Physical Medicine and Rehabilitation, Faculty of Medicine, University of British Columbia, Vancouver, BC, Canada; ${ }^{6}$ University Health Network-Toronto Rehabilitation Institute, Toronto, ON, Canada and ${ }^{7}$ Department of Physical Therapy, Faculty of Medicine, University of Toronto, Toronto, ON, Canada

Correspondence: Dr JJ Eng, Rehabilitation Research Lab, GF Strong Rehab Centre, 4255 Laurel Street, Vancouver, BC, Canada V5Z 2 G9.

E-mail: Janice.Eng@ubc.ca

Received 30 December 2015; revised 13 July 2016; accepted 19 July 2016; published online 18 October 2016 
their rehabilitation. This study will fill this gap by measuring movement repetitions during inpatient SCI rehabilitation.

Research evidence suggests that the weeks (animal studies) and months (human studies) following SCI are a critical time window for optimizing recovery, ${ }^{16-19}$ when the central nervous system is more sensitive to stimuli and experience, including exercise in the form of repetitive practice of voluntary movements. This time generally corresponds to the sub-acute inpatient rehabilitation stay. Owing to the SCI itself, any accompanying injuries, prolonged bed rest and a host of psychological sequelae, it is expected that patients may tolerate a small number of repetitions early during rehabilitation, but progress over time to challenge the neurological and musculoskeletal systems. Thus, knowing the current repetitions of activities during this period of rehabilitation and how they progress over time will provide a baseline of activity levels and set the stage for clinical trials aimed at developing interventions to enhance motor learning and improve rehabilitation outcomes.

Our aims were to (1) quantify the amount of movement repetitions (active therapist-directed movement repetitions involving voluntary movements) that patients experience for the upper extremity and lower extremity during inpatient SCI rehabilitation and (2) quantify changes in the amount of movement repetitions that patients with SCI undertake over their time in rehabilitation. To investigate our questions we completed the first study to observe PT and OT sessions and collected information on the amount of repetitions, type of activity and time spent on activities during inpatient SCI rehabilitation. We expected that movement repetitions would increase for PT and OT sessions over the SCI inpatient rehabilitation stay, commensurate with improvements in patient function. We also expected that movement repetitions for both upper and lower extremities would be low during PT and OT sessions.

\section{MATERIALS AND METHODS \\ Participants}

Patients with traumatic and non-traumatic SCI were recruited from consecutive admissions to inpatient sub-acute care at two stand-alone rehabilitation centers from November 2010 to December 2012. Non-traumatic SCI was defined as that resulting from spinal stenosis, tumor, ischemia, transverse myelitis and infection. ${ }^{20}$ Ambulatory participants were defined as those who were independently ambulatory (with or without assistive devices) at the time of discharge assessment. Patients were excluded if they had a traumatic brain injury that significantly affected content and delivery of therapy or if their length of stay in rehabilitation was projected to be $<4$ weeks as it precluded the ability to collect admission and discharge data.

Approval for this study was obtained from the university research ethics boards. All observed patients and therapists provided informed consent before therapy observation.

\section{Observed therapy sessions}

A trained observer recorded all activities that a patient performed, under the direction of a physical therapist, occupational therapist or rehabilitation assistant. The observations most often included PT and OT sessions but also supplementary sessions with rehabilitation assistants in the rehabilitation area and on the ward. As we wanted to measure typical active therapy sessions, the first measurement occurred in the second week after admission to avoid observation of sessions involving assessments. Observers recorded all PT and OT therapy activities that occurred on two days within that week. The final measurement took place in the second-last week before discharge to avoid discharge planning and re-assessment activities. Again, two days within that week were observed. Although patients were assessed at different times post injury, our choice of measurement time is a clinically relevant option as it uses specific criteria for admission to inpatient rehabilitation services and ensures that patients are ready to be engaged in intensive rehabilitation activities. To be admitted for inpatient rehabilitation from acute care, patients must fulfill the following criteria: be medically stable and benefit from a short-stay interdisciplinary rehabilitation program; be able to follow simple commands; be able to learn and recall enough information from day to day to participate; have identified specified rehabilitation goals that are attainable; and have adequate cognition, motivation, behavior and endurance to benefit from an intensive rehabilitation program. Criteria for discharge from inpatient services include having achieved goals for inpatient rehabilitation, the patient no longer making any functional gains, the patient refusing to participate in active rehabilitation, or being medically unstable.

Therapy sessions were included for observation if more than $50 \%$ of therapeutic time comprised physical rehabilitation. Therapeutic time was defined as any activity undertaken by the therapist with the goal of treating the patient and included physical rehabilitation, education, assessments and interventions designed to improve functional independence. Sessions were excluded if more than $50 \%$ of therapeutic time comprised admission or discharge assessments, equipment fitting or non-motor issues (for example, discharge planning, education). Thus, our criteria allowed us to assess therapeutic repetitions under a best-case scenario, and omitting sessions not representative of the majority of therapy sessions. Non-therapeutic time was defined as any activity that occurred but was not for treating the patient's condition, such as talking, resting, changing location or setting up for the next activity. If a session did not occur on a scheduled day or did not meet the criteria indicated above, an additional day of therapy was observed if it occurred within one week of the first day of data collection.

Standardization between trained and new observers was accomplished by an orientation where new observers were familiarized with the data collection protocol and then recorded sessions with an experienced observer. The lead investigator and the observer compared therapy observation data sheets following therapy sessions; feedback was provided, and further sessions were recorded under supervision until the data recorded were at $\geqslant 95 \%$ agreement. In addition to the lead investigator, four individuals (one physical therapist and three research assistants) observed therapy sessions over the course of the study.

During the observed therapy sessions, the observers situated themselves such that they were able to clearly see and hear the therapy session while being a distance away such that their presence did not interfere with therapy delivery. Moreover, observers did not engage the therapist or patient during the session.

To record information, the observer used a stopwatch and data collection sheets to document the type of therapy (PT or OT), repetitions, movement classification and duration of activity. Movements were classified via a taxonomy (Table 1) modified from others. ${ }^{21-23}$ The categories used in this study included the following: upper extremity (including all arm and hand movements); hand (a subset including only repetitions of the hand/wrist); lower extremity (including all lower-extremity activities); and stepping (a subset of lower extremity including only stepping on flat surfaces or ascending/ descending stairs). As we wished to include only those repetitions that contributed most to motor and functional recovery (Tables 3-5), we excluded passive movements.

On the occasion that the patient was undergoing two therapeutic activities at the same time (for example, simultaneous upper- and lower-extremity repetitions), both movements were recorded and included as therapeutic activities. In calculating therapeutic time in a session, we subtracted non-therapeutic time (for example, resting) from total therapy time to avoid the possibility of therapeutic time being longer than the actual session time.

Outside of therapy observation, patients were asked how many minutes of structured group classes they attended that day. These classes were not observed as patients were not followed outside of individual PT and OT therapy sessions. Group classes included wheelchair skills, pulleys (upper body) and hand function.

\section{Clinical outcome measures}

Clinical outcome measures were collected on a separate day within the admission and discharge data collection periods.

The Spinal Cord Independence Measure III assesses the ability of SCI patients to accomplish activities of daily living in the area of self-care, 
Table 1 Definitions of movements, units of repetition and examples for categories and subcategories

\begin{tabular}{|c|c|c|c|}
\hline Category & Definition & Definition of a single repetition & Examples \\
\hline \multicolumn{4}{|c|}{ Upper extremity } \\
\hline Total & $\begin{array}{l}\text { Any movement in which the patient attempted to or moved } \\
\text { the upper limb through a specific motion or attempted or } \\
\text { accomplished a functional task }\end{array}$ & $\begin{array}{l}\text { One movement of } 1 \text { limb from initial position and back again } \\
\text { OR one movement from initial position to desired position OR } \\
\text { one movement from one surface to another using the arms }\end{array}$ & $\begin{array}{l}\text { Dumbbell exercises, pulley } \\
\text { exercises using the arm, trans- } \\
\text { fers, mobility }\end{array}$ \\
\hline Hand only & $\begin{array}{l}\text { Any movement in which the patient attempted or moved } \\
\text { the finger(s) or wrist through a specific motion }\end{array}$ & One movement from initial position and back again & $\begin{array}{l}\text { Working on grip/dexterity, wrist } \\
\text { roller, squeezing ball, pegboard }\end{array}$ \\
\hline \multicolumn{4}{|c|}{ Lower extremity } \\
\hline Total & $\begin{array}{l}\text { Any movement in which the patient attempted or moved } \\
\text { the lower limb through a specific motion }\end{array}$ & One movement of $1 \mathrm{limb}$ from initial position and back again & $\begin{array}{l}\text { Hip abduction; knee extension; } \\
\text { hamstring curl, balance training }\end{array}$ \\
\hline Gait & $\begin{array}{l}\text { Walking overground or on a treadmill; going up and/or } \\
\text { down stairs }\end{array}$ & Each step of each foot & Walking \\
\hline
\end{tabular}

Table 2 Demographic and $\mathrm{SCl}$ information for all patients and subgroups of paraplegia and tetraplegia and ambulatory patients

\begin{tabular}{|c|c|c|c|c|}
\hline Variable & All patients & Paraplegia & Tetraplegia & Ambulatory ${ }^{\mathrm{a}}$ \\
\hline$n$ & 103 & 55 & 48 & 45 \\
\hline Gender (M/F) & $75 / 28(73 / 27)$ & $38 / 17(69 / 31)$ & $37 / 11(77 / 23)$ & $33 / 12(73 / 27)$ \\
\hline Traumatic/non-traumatic & $68 / 35(66 / 34)$ & $32 / 23(58 / 42)$ & $36 / 12(75 / 25)$ & $28 / 17(62 / 38)$ \\
\hline AIS score $(A / B / C / D)^{b}$ & 23/12/12/56 (22/12/12/54) & $12 / 6 / 9 / 28(22 / 11 / 16 / 51)$ & $11 / 6 / 3 / 28(23 / 13 / 6 / 58)$ & $1 / 2 / 0 / 42(2 / 4 / 0 / 93)$ \\
\hline Age (years) & $49 \pm 17,53,69$ & $48 \pm 18,52,68$ & $50 \pm 17,53,60$ & $50 \pm 16,54,66$ \\
\hline LOS in rehabilitation (days) & $96 \pm 46,93,225$ & $85 \pm 38,76,224$ & $110 \pm 51,116,215$ & $76 \pm 43,65,215$ \\
\hline LOS in acute care (days) & $38 \pm 38,23,170$ & $32 \pm 33,20,154$ & $46 \pm 43,30,165$ & $20 \pm 13,16,54$ \\
\hline
\end{tabular}

Abbreviations: AIS, American Spinal Injury Association Impairment Scale; LOS, length of stay; $n$, Number of patients.

Values are $n(\%)$ or mean \pm s.d., median, range.

aThe ambulatory group is composed of a subset of individuals from paraplegia and tetraplegia who were able to ambulate by the time of the discharge assessment.

${ }^{\mathrm{b}}$ Although the AIS is valid for traumatic SCI, it has not been validated in non-traumatic SCI.

respiration and sphincter management, and mobility, ${ }^{24}$ and is scored from 0 to 100 with higher scores indicating better functional independence. The measure has excellent validity and reliability. ${ }^{25}$

Ambulatory patients were assessed with the Walking Index for SCI II, designed to gauge ambulation over a $10-\mathrm{m}$ distance with ambulation aids and physical assistance. Locomotor ability is assessed on a 0-20 hierarchical scale where a lower number indicates higher impairment. This assessment shows excellent reliability and validity. ${ }^{26}$

Grip strength was tested using a hand-held Jamar Dynamometer (Nicholas MMT, Lafayette Instrument, Lafayette, IN, USA). Patients performed three maximal voluntary contractions, with at least $30 \mathrm{~s}$ of rest between trials. The trials were averaged to obtain a mean score in $\mathrm{kg}$. Measurements were taken with the patient seated, the elbow bent at $90^{\circ}$ and hand in a neutral position. This test is reliable and valid for assessing grip in healthy and hand-injured populations. ${ }^{27,28}$

The Graded Redefined Assessment of Strength, Sensibility and Prehension was used in patients with tetraplegia to evaluate muscle, sensory and grasping function. This assessment involves scoring six functional tasks and assessing upper-extremity strength and sensibility (that is, perception of sensation) of the hands; scores for each hand are summed $(0-116)$, with higher scores indicating better hand function. ${ }^{29}$ The assessment has demonstrated reliability and validity in the SCI population..$^{30}$

Demographic information was collected for age, gender, injury level (paraplegia/tetraplegia), American Spinal Injury Association Impairment Scale score, etiology (traumatic or non-traumatic) and length of stay in acute care and rehabilitation.

\section{Data analysis}

For OT and PT, therapy time and repetitions were calculated by averaging sessions occurring over two days to obtain a daily therapy value. Descriptive statistics for patient demographics are included in Table 2. Clinical outcome measures are presented in Table 3, and the therapeutic time and movement repetition data for patients with paraplegia and tetraplegia are presented in Tables 4 and 5, respectively. We also provide descriptive data for the tetraplegia group separated by complete and incomplete SCI status (Table 6), but these data are not assessed statistically because of the small size of these subsets and overlap with other analyses. In addition, we present data for those patients able to ambulate by the time of their discharge assessment because it is likely that their therapy sessions involved ambulatory goals and activities (Table 7). Wilcoxon-signed rank tests determined whether therapy times (total time, therapeutic time) and movement repetitions (total upper extremity, hand, total lower extremity, steps) changed over the rehabilitation stay from admission to discharge. Median, interquartile range, $Z$-value, and effect size $(r=Z / \sqrt{n})$ are documented (small effect size $=0.1$; medium effect size $=0.3$; large effect size $=0.5){ }^{31,32}$ For clinical outcome measures, means, standard deviations and confidence intervals for admission and discharge are documented.

Statistical software SPSS 17 (SPSS, Chicago, IL, USA) was used for the analysis. Given the number of tests employed, a Benjamini-Hochberg calculated alpha was used to correct for multiple comparisons and minimize type I error. ${ }^{33}$ On the basis of the number of comparisons, a BenjaminiHochberg alpha value of 0.008 was calculated and utilized. Reported values are medians unless stated otherwise.

\section{RESULTS}

\section{Patient demographics and clinical outcomes}

A total of 115 patients entered the study from November 2010 to December 2012 (recruitment information provided in Figure 1). Of these, we attained a discharge evaluation for 103 (90\%). Although we could not attain a discharge evaluation for 12 patients because they were discharged with insufficient notice, demographic variables for 
Table 3 Clinical outcome measures at admission and discharge from inpatient $\mathrm{SCl}$ rehabilitation

\begin{tabular}{lccc}
\hline Variable & Admission $^{\mathrm{a}}$ & Discharge $^{\mathrm{b}}$ & $95 \% \mathrm{Cl}$ \\
\hline $\begin{array}{l}\text { Paraplegia } \\
\quad\end{array}$ & & & \\
$\quad$ Grip strength (kg) & $34.8 \pm 13.5$ & $35.3 \pm 13.5$ & $-2.3,1.3$ \\
SCIM III-total & $50.7 \pm 17$ & $66.6 \pm 17.1$ & $-19.2,-12.6$ \\
& & & \\
Tetraplegia & & & \\
Grip strength (kg) & $6.4 \pm 10.6$ & $9.7 \pm 10.9$ & $-5.4,-1.3$ \\
GRASSP & $64.0 \pm 35.9$ & $77.7 \pm 34.9$ & $-19.2,-8.3$ \\
SCIM III-total & $32.7 \pm 23.8$ & $52.3 \pm 27.8$ & $-24.6,-14.6$ \\
& & & \\
Ambulatory patients & & & \\
1OMWT-comfortable (m s & & & \\
WISCI II & $0.30 \pm 0.40$ & $0.76 \pm 0.37$ & $-0.6,-0.4$ \\
SCIM III-total & $6.4 \pm 7.9$ & $15.5 \pm 5.3$ & $-11.5,-6.7$ \\
\hline
\end{tabular}

Abbreviations: 10MWT, 10 m walk test; $\mathrm{Cl}$, confidence interval; GRASSP, Graded Redefined Assessment Of Strength, Sensitivity And Prehension; SCIM III, Spinal Cord Independence

Measure III; WISCI II, Walking Index for Spinal Cord Injury II.

aSecond week after admission.

Second week before discharge.

All values are means \pm s.d.

Table 4 Patients with paraplegia: therapy time and repetitions at admission and discharge from inpatient $\mathrm{SCl}$ rehabilitation

\begin{tabular}{|c|c|c|c|c|c|}
\hline Variable & Admission ${ }^{\mathrm{a}}$ & Discharge $^{\mathrm{b}}$ & Z-value & $\mathrm{P}$-value & r-value \\
\hline \multicolumn{6}{|l|}{ Physical therapy $(n=55)$} \\
\hline Total time (min) & $54,45-59$ & $53,37-62$ & -0.60 & 0.55 & -0.06 \\
\hline Therapeutic time (min) & $32,24-39$ & $33,22-41$ & -0.36 & 0.72 & -0.03 \\
\hline Upper extremity (reps) & $7,0-55$ & $9,0-34$ & -0.91 & 0.37 & -0.09 \\
\hline Lower extremity (reps) & $30,0-155$ & $50,0-230$ & -1.93 & 0.053 & -0.18 \\
\hline \multicolumn{6}{|c|}{ Occupational therapy $(n=49)$} \\
\hline Total time (min) & $34,12-48$ & $0,0-31$ & -3.48 & $0.001 *$ & -0.35 \\
\hline Therapeutic time (min) & $17,7-25$ & $0,0-17$ & -3.34 & $0.001^{*}$ & -0.34 \\
\hline Upper extremity (reps) & $0,0-4$ & $0,0-0$ & -1.63 & 0.10 & -0.17 \\
\hline Lower extremity (reps) & $0,0-0$ & $0,0-0$ & -0.73 & 0.46 & -0.07 \\
\hline
\end{tabular}

Abbreviations: $n$, number of patients; $r$, effect size.

asecond week after admission.

becond week before discharge.

All values are median, Q1-Q3. ${ }^{*} P \leqslant 0.008$ (Benjamini-Hochberg-corrected significance level).

these patients (not reported) were similar to those included for analyses. Demographic information is provided in Table 2. From the 103 patients in this study, we observed 561 PT sessions and 347 OT sessions. Some patients did not engage in any session over the observed week that was focused on physical activities and were assigned a value of zero repetitions. This occurred for four patients before discharge from PT, for 22 patients after OT admission and for 42 patients before OT discharge.

There were clinically meaningful improvements for all clinical outcome measures except grip strength for individuals with paraplegia (Table 3).

\section{Changes in therapy time}

Total therapy session time and therapeutic time did not change during PT sessions over the rehabilitation stay, but did during OT sessions for individuals with paraplegia who experienced a reduction in both these variables (Total time: 34 versus $0 \mathrm{~min}$; Therapeutic time: 17 versus
Table 5 Patients with tetraplegia: therapy time and repetitions at admission and discharge from inpatient $\mathrm{SCl}$ rehabilitation

\begin{tabular}{llllll}
\hline Variable & Admission & Discharge & Z-value & P-value & r-value \\
\hline Physical therapy $(\mathrm{n}=48)$ & & & & & \\
$\quad$ Total time (min) & $53,44-62$ & $52,37-62$ & -0.46 & 0.64 & -0.05 \\
$\quad$ Therapeutic time (min) & $31,23-38$ & $31,24-43$ & -1.54 & 0.12 & -0.16 \\
Upper extremity (reps) & $11,0-53$ & $13,0-68$ & -1.14 & 0.26 & -0.12 \\
Hand (reps) & $0,0-0$ & $0,0-0$ & -0.67 & 0.50 & -0.07 \\
Lower extremity (reps) & $13,0-127$ & $33,0-215$ & -0.83 & 0.41 & -0.09 \\
& & & & & \\
Occupational therapy (n= & $43)$ & & & & \\
Total time (min) & $44,26-54$ & $30,0-46$ & -2.29 & 0.022 & -0.25 \\
Therapeutic time (min) & $27,13-35$ & $18,0-31$ & -2.50 & 0.012 & -0.27 \\
Upper extremity (reps) & $31,0-127$ & $2,0-34$ & -2.97 & $0.003 *$ & -0.32 \\
Hand (reps) & $7,0-57$ & $0,0-15$ & -3.12 & $0.002 *$ & -0.34 \\
Lower extremity (reps) & $0,0-0$ & $0,0-0$ & -0.17 & 0.87 & -0.02 \\
\hline
\end{tabular}

Abbreviations: $n$, number of patients; $r$, effect size.

asecond week after admission.

bSecond week before discharge.

All values are median, Q1-Q3. ${ }^{*} P \leqslant 0.008$ (Benjamini-Hochberg-corrected significance level).

0 min) (Table 4). For all patients, total time in therapy for PT and OT sessions combined amounted to a median $1.6 \mathrm{~h}$ at admission and $1.1 \mathrm{~h}$ at discharge, and on average $60 \%$ of total time was classified as therapeutic time.

\section{Changes in upper-extremity repetitions}

Total daily upper-extremity repetitions can be found in Figure 2. Upper-extremity repetitions were primarily undertaken in OT. More specifically, for patients with tetraplegia, upper-extremity repetitions decreased significantly from 31 to 2 in OT (Table 5). Hand repetitions (Table 5), a subset of upper-extremity repetitions, were low in OT sessions (7 repetitions) and decreased significantly over the rehabilitation stay (0 repetitions). During OT, repetitions were notably higher for tetraplegic individuals with motor incomplete injury (Table 6).

In the subset of individuals with paraplegia (Table 4), upper-extremity repetitions were low after admission (7 in PT sessions and negligible in OT sessions) and did not change over the rehabilitation stay.

\section{Changes in lower-extremity repetitions}

Total daily lower-extremity repetitions can be found in Figure 2 . Lower-extremity repetitions were primarily undertaken in PT. For participants with paraplegia (Table 4) and tetraplegia (Table 5), lowerextremity repetitions did not exceed 30 repetitions after admission in PT but did not change significantly over the rehabilitation stay, and were higher for motor incomplete individuals with tetraplegia (Table 6) and paraplegia compared with those with motor complete injury.

For patients who were ambulating by the time of their discharge assessment (Table 7), the values for lower-extremity repetitions (143) and steps (51) after admission in PT sessions did not change significantly over the rehabilitation stay.

\section{Participation in group classes}

The average time spent in group classes was 12 min after admission and $15 \mathrm{~min}$ before discharge. 
Table 6 Patients with tetraplegia: therapy time and repetitions separating for motor complete and motor incomplete injury

\begin{tabular}{|c|c|c|c|c|c|}
\hline \multirow[t]{2}{*}{ Variable } & \multicolumn{3}{|c|}{ Motor complete } & \multicolumn{2}{|c|}{ Motor Incomplete } \\
\hline & Admission ${ }^{a}$ & & Discharge $^{b}$ & Admission ${ }^{a}$ & Discharge $^{b}$ \\
\hline$n$ & & 17 & & & \\
\hline Total time (min) & $52,45-61$ & & $54,44-66$ & $54,43-63$ & $50,34-62$ \\
\hline Therapeutic time (min) & $26,23-37$ & & $32,24-39$ & $33,22-41$ & $30,24-44$ \\
\hline Lower extremity (reps) & $0,0-0$ & & $0,0-0$ & $82,21-207$ & $145,34-342$ \\
\hline \multicolumn{6}{|l|}{ Occupational therapy } \\
\hline$n$ & & 16 & & & \\
\hline Total time (min) & $28,15-49$ & & $23,0-44$ & $45,35-56$ & $37,12-46$ \\
\hline
\end{tabular}

Abbreviation: $n$, number of patients.

asecond week after admission.

bSecond week before discharge.

All values are median, Q1-Q3.

Table 7 Ambulatory patients: therapy time and repetitions at admission and discharge from inpatient $\mathrm{SCl}$ rehabilitation

\begin{tabular}{|c|c|c|c|c|c|}
\hline Variable & Admission ${ }^{a}$ & Discharge $^{\mathrm{b}}$ & Z-value & P-value & r-value \\
\hline \multicolumn{6}{|l|}{ Physical therapy $(n=45)$} \\
\hline Total time (min) & $54,45-61$ & $53,37-68$ & -0.21 & 0.83 & -0.02 \\
\hline Lower extremity (reps) & $143,65-300$ & $218,114-381$ & -1.03 & 0.30 & -0.11 \\
\hline Steps (reps) & $51,0-176$ & $115,21-313$ & -1.19 & 0.23 & -0.13 \\
\hline Therapeutic time (min) & $24,10-33$ & $16,0-25$ & -2.24 & 0.025 & -0.25 \\
\hline Lower extremity (reps) & $0,0-3$ & $0,0-0$ & -0.53 & 0.59 & -0.06 \\
\hline Steps (reps) & $0,0-0$ & $0,0-0$ & 0.00 & 1.0 & 0.00 \\
\hline
\end{tabular}

Abbreviations: $n$, number of patients; $r$, effect size.

asecond week after admission.

bSecond week before discharge.

All values are median, Q1-Q3. * $P \leqslant 0.008$ (Benjamini-Hochberg-corrected significance level).

\section{DISCUSSION}

\section{Amount of movement repetitions}

Our primary finding is that repetitions are notably low during rehabilitation. Individuals with motor incomplete tetraplegia, who had the highest amount of upper-extremity repetitions, reached a median value of 115 during PT and OT sessions combined, with all other groups not reaching a quarter of this amount. Ambulatory individuals, who had the highest amount of lower-extremity repetitions and steps, did not exceed a respective 218 and 115 repetitions during combined PT and OT sessions measured at discharge. These repetition values are markedly lower than those that have been reported to be necessary for optimizing neuroplastic changes. ${ }^{14,15,34}$ These repetitions are also low for musculoskeletal or endurance functions. For example, a typical wheeling push frequency is one push per second, ${ }^{35}$ and the twenty upper-extremity repetitions (median total for PT and OT) measured in our patients with paraplegia would hypothetically allow one to wheel for a fraction of

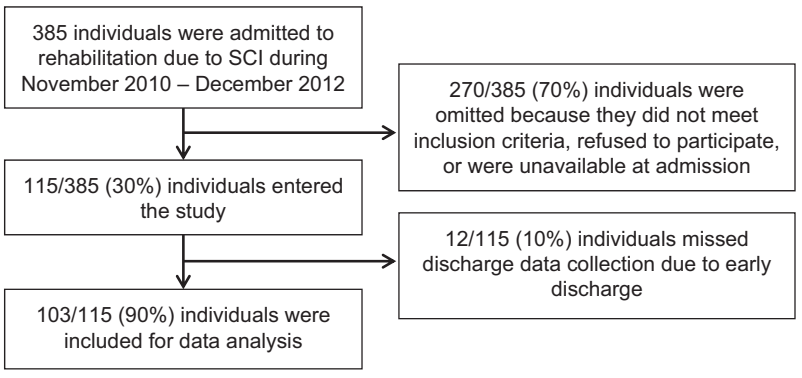

Figure 1 Flow diagram of recruitment to the study.

a minute. These repetition levels would not be sufficient to strengthen the upper extremities to prevent overuse injuries or develop upperextremity endurance for wheeling. Similarly, for ambulatory patients, the 115 steps (total for PT and OT) that we observed at the discharge 


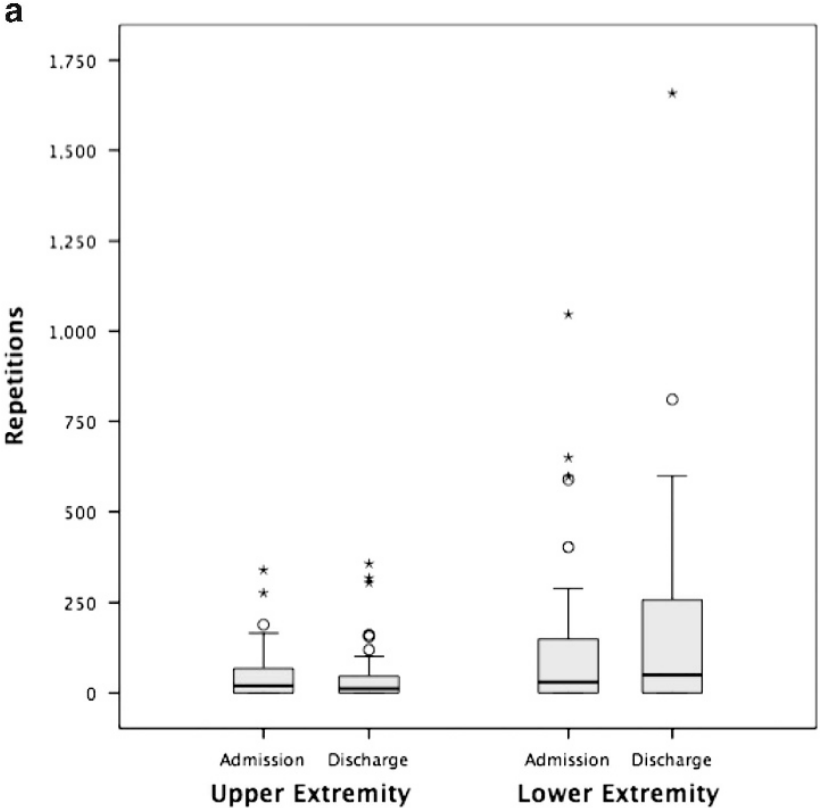

b

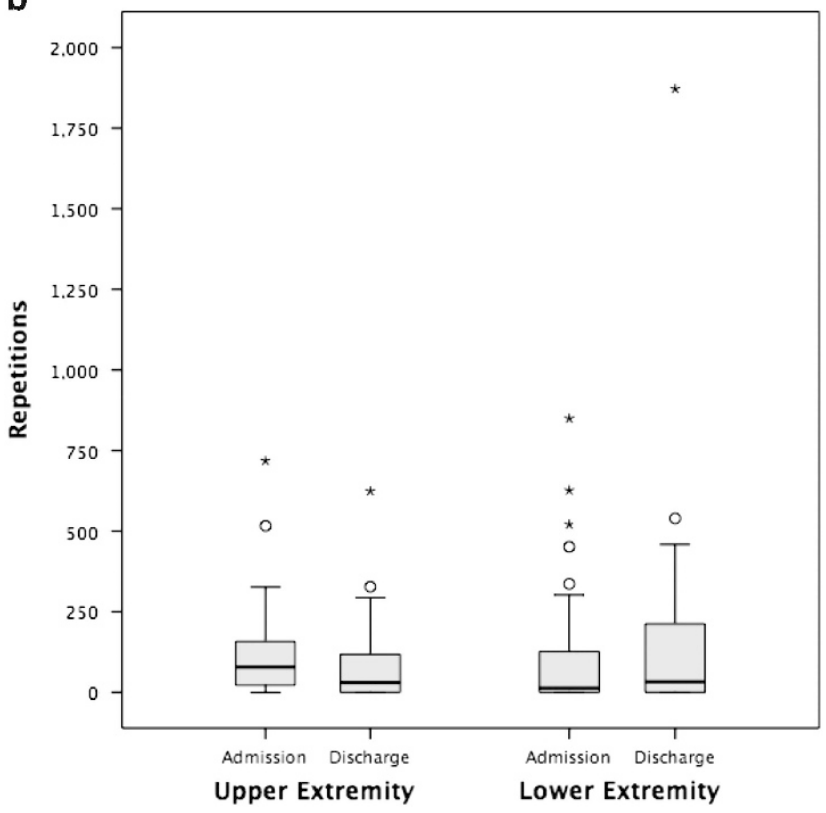

Figure 2 Daily repetitions for physical therapy and occupational therapy combined. (a) Group with paraplegia. (b) Group with tetraplegia. Values are median and interquartile range.

gait speed $\left(0.76 \mathrm{~m} \mathrm{~s}^{-1}\right)$ with a typical short step length $(0.5 \mathrm{~m})$ would hypothetically result in only $1.3 \mathrm{~min}$ of walking practice over $58 \mathrm{~m}$, which is not sufficient for any community ambulation activity.

In the animal literature, movement repetitions are acutely challenged where success rates of only $60 \%$ are experienced for reaching tasks ${ }^{10}$ and likely contribute to the positive effects of task-specific training. ${ }^{10,36}$ We know from observation that the large majority of patients were able to complete all repetitions prescribed. It is possible that individuals with SCI could perform more difficult exercises to improve gains during rehabilitation stay. However, it is likely that therapists prescribe exercise that is challenging yet at the same time meets with successful execution as failed repetitions could be demotivating to a patient and, in some activities, potentially injurious.

\section{Changes in movement repetitions}

For the most part, little change in repetitions occurred over the rehabilitation stay. This may be attributed, on the one hand, to patients who met their therapeutic goals earlier in their stay and then focused on non-motor activities as they approached discharge time and, on the other hand, to patients who were not able or motivated to undertake larger amounts of repetitions over time because of factors such as fatigue, pain, or depressive symptoms. It is also possible that therapists did not provide sufficient time for patients to engage in movement repetitions during therapy, and this could be the result of limited time combined with numerous rehabilitation goals outside of practicing movement repetitions. Given that $61 \%$ of patients had motor incomplete injuries, it would appear that patients could have benefited from further motor training, but other priorities left little time for these activities.

Although much neuroplasticity research measures or manipulates intensity through movement repetitions (for example, number of reaches for a food pellet), the reality of inpatient SCI rehabilitation is more complex. In addition to repetitions, the intensity of an activity may be increased by adding a greater load or by selecting a more difficult movement. For example, 30 repetitions may have been done with a $2 \mathrm{~kg}$ weight after admission and with $5 \mathrm{~kg}$ before discharge. Although it is possible that increases in intensity through means other than greater repetitions may hinder the detection of significant differences from admission to discharge, the field of research investigating the role of rehabilitation in neuroplasticity ${ }^{11-15,34}$ is dominated by manipulation of dose through repetitions. We have shown that repetitions are low at both time points.

\section{Therapeutic versus non-therapeutic time}

It is common for clinical research to use hours of therapy as an independent variable when evaluating outcomes, ${ }^{2-8}$ and health-care guidelines often use this metric. ${ }^{37}$ However, we found that approximately 40 percent of a session was non-therapeutic time (for example, sling transfers, setting up the next activity): activities not therapeutic in themselves but necessary for delivering therapy. If appropriate therapeutic guidelines are to be made for public policy decisions, the actual patient time spent engaged in the therapeutic interventions needs to be considered.

\section{Changes in clinical outcomes}

Despite repetitions being notably below repetition volumes seen in the human and animal motor-learning literature, patients experienced improvements on clinical outcome measures, reflecting a combination of natural recovery and effects from the rehabilitation process. Nevertheless, improvement is not the same as optimization, and the finding that therapy repetitions are vastly fewer than task-specific training protocols suggests that methods to increase repetitions would move us toward optimizing clinical outcomes. However, as mentioned earlier, therapy time cannot be solely dedicated to high-repetition taskspecific training for gait or reaching, as numerous other therapeutic goals such as addressing spastic musculature or pain take up the patient's time, which is already limited as approximately one-third of sessions is spent on non-therapeutic activities (for example, repositioning) that are necessary for delivering therapy. One alternative that has been successful in the stroke inpatient setting ${ }^{38,39}$ is to accumulate repetitions outside of therapy time with the support of caregivers or 
rehabilitation assistants. The same potential may exist in therapy for individuals with SCI.

\section{Limitations}

We did not monitor repetitions that occurred outside of PT- and OTdirected activities - for example, during group classes and activities of daily living. However, group class time was low, with a median (Q1-Q3) value of 0 (0-30) $\mathrm{min}$ per day; almost two-thirds of individuals did not engage in group classes during measurement days. Also it is likely that the active time was less, given the nature of group classes being more social, and set-up time required for multiple participants.

Although nine persons with concomitant traumatic brain injury were included, we excluded four individuals with severe traumatic brain injury whose therapy had a focus on cognitive rather than on physical rehabilitation. We also excluded nine individuals with a length of stay shorter than four weeks. Although the number of participants who were excluded for these reasons is small, this may limit how well our sample represents the true breadth of the clinical presentation of SCI.

It is possible that social desirability bias resulted in the therapists undertaking more repetitions than usual. We believe we minimized this risk as therapists were specifically asked to deliver therapy as they ordinarily would and to not involve or communicate with the observer during the observed session. In addition, it is difficult to conceive that therapists would change their practice for the two years for which data were being collected.

\section{CONCLUSION}

The amount of movement practice that occurs during inpatient SCI therapy is notably low, and does not appear to progress over time. The implication is that the stimuli applied during inpatient stay may not be adequate to maximize the musculoskeletal or neural changes needed to promote optimal function after SCI.

We recommend that future research investigate patient perception of physical activity intensity during rehabilitation.

\section{DATA ARCHIVING}

There were no data to deposit.

\section{CONFLICT OF INTEREST}

The authors declare no conflict of interest.

\section{ACKNOWLEDGEMENTS}

DZ is supported by a Doctoral Frederick Banting and Charles Best Canada Graduate Scholarship from the Canadian Institutes of Health Research. JJE acknowledges salary support from the Canada Research Chair Program. This research is supported by the Canadian Institutes of Health Research. We thank the research assistants for their work, especially Erika Brown, Jenna Homer, and Amanda Mow, and the rehabilitation centers, therapists and patients who participated in this study.

1 Whiteneck G, Gassaway J, Dijkers M, Backus DD, Charlifue S, Chen D et al. Inpatient treatment time across disciplines in spinal cord injury rehabilitation. J Spinal Cord Med 2011; 34: 133-148.

2 Teeter L, Gassaway J, Taylor S, LaBarbera J, McDowell S, Backus DD et al. Relationship of physical therapy inpatient rehabilitation interventions and patient characteristics to outcomes following spinal cord injury: the SCIRehab project. J Cord Med 2012; 35: 503-526.

3 Ozelie R, Gassaway J, Buchman E, Thimmaiah D, Heisler L, Cantoni K et al. Relationship of occupational therapy inpatient rehabilitation interventions and patient characteristics to outcomes following spinal cord injury: the SCIRehab project. J Spinal Cord Med 2012; 35: 527-546.

4 Heinemann AW, Hamilton B, Linacre JM, Wright BD, Granger C. Functional status and therapeutic intensity during inpatient rehabilitation. Am J Phys Med Rehabil 1995; 74: 315-326.

5 van Langeveld SA, Post MW, van Asbeck FW, Gregory M, Halvorsen A, Rijken $\mathrm{H}$ et al. Comparing content of therapy for people with a spinal cord injury in postacute inpatient rehabilitation in Australia, Norway, and the Netherlands. Phys Ther 2011; 91: 210-224.

6 van Langeveld SA, Post MW, van Asbeck FW, Horst ter P, Leenders J, Postma K et al. Contents of physical therapy, occupational therapy, and sports therapy sessions for patients with a spinal cord injury in three Dutch rehabilitation centres. Disabil Rehabil 2011; 33: 412-422.

7 Taylor-Schroeder S, LaBarbera J, McDowell S, Zanca JM, Natale A, Mumma S et al. The SCIRehab project: treatment time spent in $\mathrm{SCl}$ rehabilitation. Physical therapy treatment time during inpatient spinal cord injury rehabilitation. J Spinal Cord Med 2011; 34: 149-161.

8 Foy T, Perritt G, Thimmaiah D, Heisler L, Offutt JL, Cantoni K et al. The SCIRehab project: treatment time spent in $\mathrm{SCl}$ rehabilitation. Occupational therapy treatment time during inpatient spinal cord injury rehabilitation. J Spinal Cord Med 2011; 34: $162-175$.

9 Edgerton VR, Tillakaratne NJK, Bigbee AJ, de Leon RD, Roy RR. Plasticity of the spinal neural circuitry after injury. Annu Rev Neurosci 2004; 27: 145-167.

10 Girgis J, Merrett D, Kirkland S, Metz GAS, Verge V, Fouad K. Reaching training in rats with spinal cord injury promotes plasticity and task specific recovery. Brain 2007; 130: 2993-3003.

11 Beekhuizen KS, Field-Fote EC. Massed practice versus massed practice with stimulation: effects on upper extremity function and cortical plasticity in individuals with incomplete cervical spinal cord injury. Neurorehabil Neural Repair 2005; 19: 33-45.

12 Hoffman LR, Field-Fote EC. Functional and corticomotor changes in individuals with tetraplegia following unimanual or bimanual massed practice training with somatosensory stimulation: a pilot study. J Neurol Phys Ther 2010; 34: 193-201.

13 Behrman AL, Bowden MG, Nair PM. Neuroplasticity after spinal cord injury and training: an emerging paradigm shift in rehabilitation and walking recovery. Phys Ther 2006; 86: 1406-1425.

14 de Leon RD, Hodgson JA, Roy RR, Edgerton VR. Locomotor capacity attributable to step training versus spontaneous recovery after spinalization in adult cats. J Neurophysiol 1998; 79: 1329-1340.

15 Lovely RG, Gregor RJ, Roy RR, Edgerton VR. Effects of training on the recovery of fullweight-bearing stepping in the adult spinal cat. Exp Neurol 1986; 92: 421-435.

16 Battistuzzo CR, Callister RJ, Callister R, Galea MP. A systematic review of exercise training to promote locomotor recovery in animal models of spinal cord injury. J Neurotrauma 2012; 29: 1600-1613.

17 Norrie BA, Nevett-Duchcherer JM, Gorassini MA. Reduced functional recovery by delaying motor training after spinal cord injury. J Neurophysiol 2005; 94: 255-264.

18 Harkema S, Gerasimenko Y, Hodes J, Burdick J, Angeli C, Chen Y et al. Effect of epidural stimulation of the lumbosacral spinal cord on voluntary movement, standing, and assisted stepping after motor complete paraplegia: a case study. Lancet 2011; 377: 1938-1947.

19 Winchester P, Smith P, Foreman N, Mosby JM, Pacheco F, Querry R et al. A prediction model for determining over ground walking speed after locomotor training in persons with motor incomplete spinal cord injury. J Spinal Cord Med 2009; 32: 63-71.

20 McKinley WO, Seel RT, Hardman JT. Nontraumatic spinal cord injury: incidence, epidemiology, and functional outcome. YAPMR 1999; 80: 619-623.

21 Ozelie RR, Sipple CC, Foy TT, Cantoni KK, Kellogg KK, Lookingbill JJ et al. SCIRehab project series: the occupational therapy taxonomy. J Spinal Cord Med 2009; 32: 283-297.

22 Natale A, Taylor S, LaBarbera J, Bensimon L, McDowell S, Mumma SL et al. SCIRehab project series: the physical therapy taxonomy. J Spinal Cord Med 2009; 32: 270-282.

23 Lang CE, MacDonald JR, Reisman DS, Boyd L, Kimberley TJ, Schindler-Ivens SM et al. Observation of amounts of movement practice provided during stroke rehabilitation. YAPMR 2009; 90: 1692-1698.

24 Catz A, Itzkovich M, Tesio L, Biering-Sorensen F, Weeks C, Laramee MT et al. A multicenter international study on the Spinal Cord Independence Measure, version III: Rasch psychometric validation. Spinal Cord 2007; 45: 275-291.

25 Itzkovich M, Gelernter I, Biering-Sorensen F, Weeks C, Laramee MT, Craven BC et al. The spinal cord independence measure (SCIM) version III: reliability and validity in a multi-center international study. Disabil Rehabil 2007; 29: 1926-1933.

26 Burns AS, Delparte JJ, Patrick M, Marino RJ, Ditunno JF. The reproducibility and convergent validity of the walking index for spinal cord injury (WISCI) in chronic spinal cord injury. Neurorehabil Neural Repair 2011; 25: 149-157.

27 Mathiowetz V, Weber K, Volland G, Kashman N. Reliability and validity of grip and pinch strength evaluations. J Hand Surg Am 1984; 9: 222-226.

28 Bohannon RW, Schaubert KL. Test-retest reliability of grip-strength measures obtained over a 12-week interval from community-dwelling elders. J Hand Ther 2005; 18: 426-427 -quiz428.

29 Rudhe C, Van Hedel HJA. Upper extremity function in persons with tetraplegia: relationships between strength, capacity, and the spinal cord independence measure. Neurorehabil Neural Repair 2009; 23: 413-421.

30 Kalsi-Ryan S, Beaton D, Curt A, Duff S, Popovic MR, Rudhe C et al. The graded redefined assessment of strength sensibility and prehension: reliability and validity. J Neurotrauma 2012; 29: 905-914. 
31 Rosenthal R. Parametric measures of effect size. In: Cooper H, Hedges LV (eds). The Handbook of Research Synthesis. Russell Sage Foundation: New York, NY, USA, 1994.

32 Cohen J. Statistical Power Analysis for the Behavioral Sciences, 2nd edn. Lawrence Erlbaum Associates: Hillsdale, NJ, USA, 1988.

33 Benjamini Y, Hochberg Y. Controlling the false discovery rate: a practical and powerful approach to multiple testing. J R Stat Soc Ser B 1995; 57: 289-300.

34 Cha J, Heng C, Reinkensmeyer DJ, Roy RR, Edgerton VR, de Leon RD. Locomotor ability in spinal rats is dependent on the amount of activity imposed on the hindlimbs during treadmill training. J Neurotrauma 2007; 24: 1000-1012.

35 Cowan RE, Boninger ML, Sawatzky BJ, Mazoyer BD, Cooper RA. Preliminary outcomes of the SmartWheel Users' Group database: a proposed framework for clinicians to objectively evaluate manual wheelchair propulsion. Arch Phys Med Rehabil 2008; 89: 260-268.
36 Weishaupt N, Li S, Di Pardo A, Sipione S, Fouad K. Synergistic effects of BDNF and rehabilitative training on recovery after cervical spinal cord injury. Behav Brain Res 2013; 239: 31-42.

37 Centers for Medicare \& Medicaid Services. Medicare Benefit Policy Manual [Internet]. www.cms.gov. www.cms.gov, 2014. Available from http://www.cms.gov/Regulationsand-Guidance/Guidance/Manuals/downloads/bp102c01.pdf. Accessed on 7 March 2014.

38 Harris JE, Eng JJ, Miller WC, Dawson AS. A self-administered Graded Repetitive Arm Supplementary Program (GRASP) improves arm function during inpatient stroke rehabilitation: a multi-site randomized controlled trial. Stroke 2009; 40: 2123-2128.

39 Connell LA, McMahon NE, Simpson LA, Watkins CL, Eng JJ. Investigating measures of intensity during a structured upper limb exercise program in stroke rehabilitation: an exploratory study. Arch Phys Med Rehabil 2014; 95: 2410-2419. 